\title{
Study on Multi-pulse Laser Detection of Moving Target under Low SNR
}

\author{
Sun Junling ${ }^{1, a}$, Pang Dongdong ${ }^{2, b}$, Sun Guangmin ${ }^{1, c}$, Ma Pengge $^{3, d}$ \\ ${ }^{1}$ The Information Department of Beijing University of Technology, Beijing 100124, China \\ ${ }^{2}$ School of Information Engineering, Zhengzhou University, Zhengzhou, Henan 450001, China \\ ${ }^{3}$ School of electronic and communication engineering, Zhengzhou University of Aeronautics, \\ Zhengzhou, Henan 450015, China \\ asunjunling@emails.bjut.edu.cn, b813244125@qq.com, 'gmsun@emails.bjut.edu.cn, \\ dmapenge@163.com
}

Keywords: Moving target; Multi-pulse detection; Laser radar; SNR; Range finder

Abstract: This paper presents a multi-pulse laser radar system and target detection algorithm for motion target detection. In this paper, the system of multi pulse lidar is introduced firstly, and the detection performance of the target range is analyzed by combining the lidar equation. Then, aiming at the detection of the moving target, the influence of the target tangential velocity and the radial velocity on the multi-pulse echo accumulation is deduced, and the conditions of the effective accumulation of the multi-pulse echo are given. The constraints of the multi-pulse laser radar target detection system on the parameters such as the irradiation frequency and the emission pulse width are pointed out. Then, a multi-pulse laser target detection algorithm and its implementation steps are proposed, and the simulation results are given. The experimental results show the effectiveness of multi-pulse laser for target detection under low SNR.

\section{Introduction}

At present, the remote moving target detection exists millimeter wave microwave radar, laser ranging and so on. How to implement the high-precision precise positioning of the remote dynamic target becomes the research hotspot of the moving target detection. Millimeter-wave radar detection has the problem of low accuracy, laser ranging often measure the stationary target, it is necessary to study the laser precision detection and positioning of the remote moving target. As the phase laser ranging measurement near, and the need for cooperation goals, the use of pulse ranging method to become the first choice for remote target laser detection. Single-pulse laser ranging requires a large amount of laser energy, so the development of low-emission power of the remote pulse laser rangefinder is the direction of development. Based on the multi-pulse laser emission system, the multi-pulse echo is accumulated under low SNR, and the target action distance is improved under the condition of low emission peak power. In this paper, the detection performance of the target range is analyzed by using the lidar equation, and the effect of the tangential velocity and radial velocity on the target echo accumulation is deduced, the condition of effective accumulation of multi pulse echo of moving targets is presented. The experimental results show that the multi pulse laser is effective for target detection under low SNR.

\section{Multi-pulse laser radar}

The multi-pulse laser radar emits a series of light pulses each time, and each process of detection and processing is controlled by a relatively large system clock to keep the transmit and receive 
synchronization. Timing diagram is shown in Fig.1:

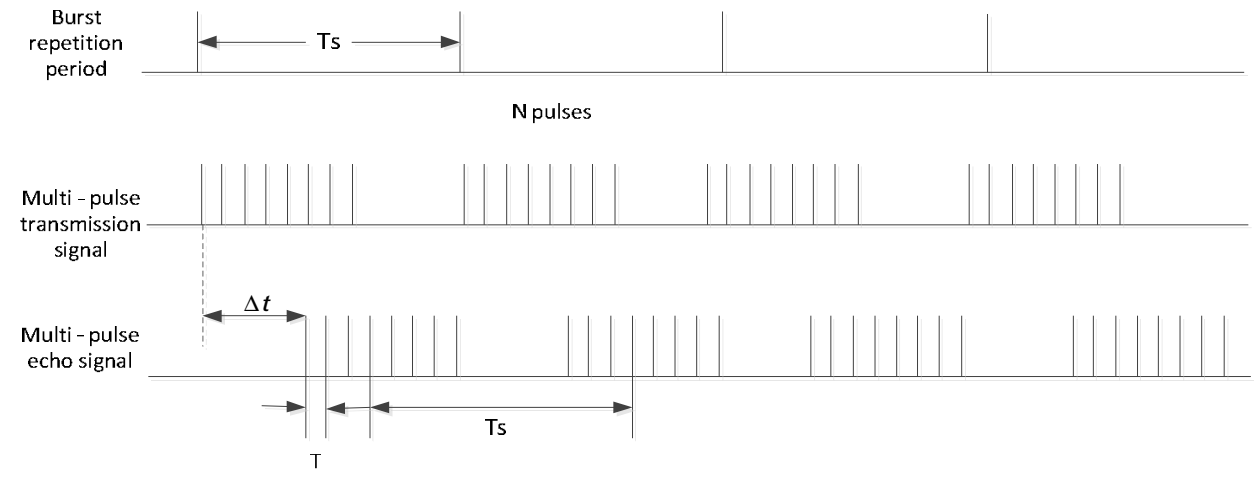

Fig.1 Multi-pulse signal timing diagram

In the system cycle, each pulse in each pulse sequence measures the same target. Correspondingly, the delay of each pulse in the echo is the same, that is, the intensity of the pulse and the distance information are the same, In the subsequent processing, the signal can be extracted from the noise by accumulating the way.

For extended targets, lidar equations:

$P_{\mathrm{det}}=\frac{P_{t} \cdot \rho_{\mathrm{exp}} \cdot D_{R}^{2}}{4 R^{2}} \tau_{a}^{2} \tau_{0}$

Where $P_{t}$ is the peak power of the emitted light pulse, $P_{\text {det }}$ is the peak power of the received detector, $D_{R}$ antenna effective aperture diameter, $\tau_{0}$ is the transmittance of the laser radar receiver optical path system $\rho_{\text {exp }}$ is the average reflection coefficient of the extended target.

The laser range of the extended target can be expressed as:

$$
R=\left[\frac{P_{t} \cdot \rho_{\exp } \cdot D_{R}^{2}}{4 P_{\mathrm{det}}} \tau_{a}^{2} \tau_{0}\right]^{\frac{1}{2}}
$$

Direct detection of laser radar is generally non-coherent accumulation, m pulse accumulation can be received to the voltage $S N R$ increased to the original $\sqrt{N}$ times. When the echo signal is weakened due to the increase of the detection distance and the SNR is lower than 1, the SNR can meet the requirement of detection by multi-pulse accumulation, which is equivalent to the operating range of the radar system.

In practical applications, the current signal output by the detector can be converted into a voltage signal through the preamplifier circuit. The echo $S N R$ can be expressed as the ratio of the maximum value of the target echo pulse to the RMS value of the echo noise:

$$
S N R=\frac{V_{\max }}{R M S}
$$

\section{Detection of moving target by multi-pulse laser radar}

Set the horizontal dimension of the target $\mathrm{d}$, the repetition period of the signal $\mathrm{T}$, the pulse width $\mathrm{r}$, and the repetition frequency $f=1 / T$. The velocity of the target is $V$. As shown in Fig.2, the target is 
decomposed: Decomposed into tangential velocity $V_{t}$, radial velocity $V_{r}$. Tangential velocity $V_{t}$ : Along the speed component perpendicular to the direction of the radar and the target connection. Radial velocity $V_{r}$ : The velocity component in the direction of the radar and the target line.

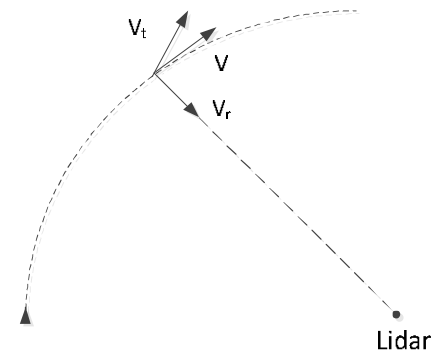

Fig.2 Target speed decomposition

\section{The effect of the tangential velocity on the accumulation}

Due to the tangential velocity $V_{t}$ such that the displacement of the target relative to the center of the laser spot is not the same when each pulse is hit on the target, so the lidar of the lid (as opposed to each pulse) is constantly changing. After a certain accumulation, the target deviates completely from the range of the radar, at this time, the back of the pulse has been unable to illuminate the target, no contribution to the accumulation, but the horizontal speed almost does not affect the time interval between adjacent pulses.

Set the cumulative number of $N$, if $N$ all the effective accumulation of pulses, each pulse must be hit on the target. When the target is regarded as the expansion target, we target the size of the target as a target to move away from the spot distance, equal to $L$ :

$$
N \cdot T \leq \frac{L}{V_{t}}
$$

$f \geq \frac{N \cdot V_{t}}{L}$

\section{The effect of the radial velocity on accumulation}

The time interval variation of the adjacent echo pulses caused by radial motion is $\Delta t$,

$$
\Delta T=\frac{2 \cdot V_{t} \cdot T}{c}
$$

Due to the radial velocity $V_{r}$, so that different pulse echo time changes, the interval of the adjacent pulse is not equal, after a certain accumulation, the subsequent pulse through a predetermined delay can not coincide with the front of the pulse, can't be carried out accumulation. But the intensity of each pulse is the same. If $N$ pulses can be effectively accumulated, the following equation should be satisfied:

$$
N \cdot \Delta T=\frac{2 \cdot N \cdot V_{r} \cdot T}{c} \leq \tau
$$




$$
f \geq \frac{2 \cdot N \cdot V_{r}}{c \cdot \tau}
$$

\section{Multi-pulse laser radar target detection algorithm}

The multi pulse echo signal can be accumulated to meet the above conditions, and the accumulated echo signal can be used for laser target detection. The maximum point detected in the current echo is not necessarily the real target. Therefore, by aiming the potential target of multi-frame echo, the target misjudgment can be avoided. The specific multi-pulse laser target algorithm is as follows:

\section{Step1: Target echo signal accumulation}

The multi-pulse echo signal is accumulated, you can get $\sqrt{N}$ times the SNR gain.

\section{Step2: Detection threshold delineation}

When the number of the thresholds in the echo data exceeds 10, the threshold is increased and the comparison is made until the number of the echo data is larger than the number of the thresholds 2 to 10 , The location of the threshold data is stored in the potential target array.

\section{Step3: Create a potential target chain}

Mark all potential targets that are detected and write down their characteristic values such as their intensity values, position values, and confidence levels, thus creating a potential target chain.

\section{Step4: Multi-frame correlation detection}

The target information of the current frame and the target information of the previous frame are correlated with the characteristics such as intensity, position and speed. When the signal is strong, the confidence is 2 , otherwise the confidence is 1 . When the confidence of the target reaches a certain value, the goal is credible and can be identified as a true goal.

\section{Step5: Target chain update}

If the target does not match successfully, it is removed from the target chain. Create a new record in the target chain for the new area. When the confidence level of a potential target in the target chain reaches the requirement, the target is captured and tracked.

\section{Step6: Target lost recapture}

Target loss is based on confidence. Set the confidence assigned to 10 after the capture target, tracking success once a confidence plus 1 , the maximum increase to 15 , the match is not successful minus 1 , confidence down to 7 , then the target is lost, transfer to the capture target phase to recapture the target.

\section{Simulation experiment:}

Fig.3 shows the average detection probability SNR. Tab.1 shows the Average acquisition time under different SNR. It can be seen that the SNR reduced to 1.2 is still valid.

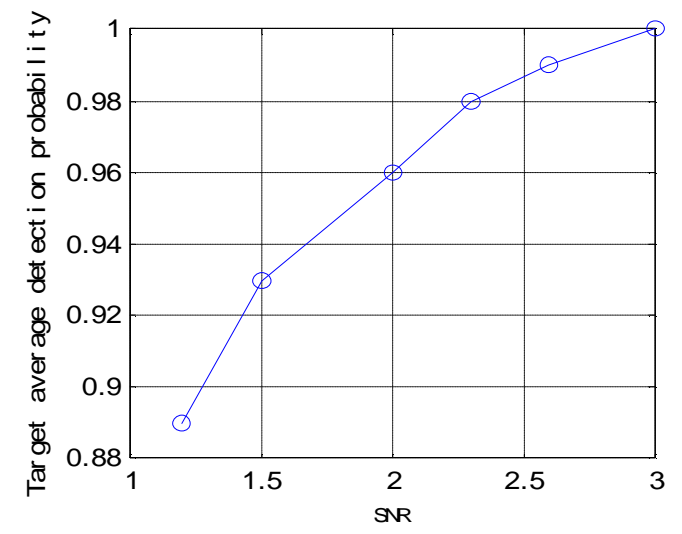

Fig.3 Average detection probability under different SNR 
Tab.1 Average acquisition time under different SNR

\begin{tabular}{lllllll}
\hline Original SNR & 1.2 & 1.5 & 2 & 2.3 & 2.6 & 3 \\
\hline Target mean acquisition time $(\mathrm{S})$ & 3 & 3 & 2 & 2 & 2 & 1 \\
\hline
\end{tabular}

\section{Conclusions}

At present, there are few domestic and international researches on multi pulse lidar. In this paper, a new radar system is proposed. The multi-pulse laser target detection algorithm and implementation steps are proposed. Based on the characteristics of multi pulse laser radar, the relationship between the pulse accumulation and the range of the target, the influence of the target velocity on the pulse accumulation is simulated. The results show: The multi-pulse echo signal satisfying the effective accumulation condition of the moving target is accumulated at the low signal-to-noise ratio, and the accumulated echo signal can be used for laser target detection and the target can be effectively detected.

\section{Acknowledgements}

This work was financially supported by Henan Province Science and Technology Project (152102210139) and Henan University Science and Technology Innovation Team (17IRTSTHN014).

\section{References}

[1] Pengge Ma, Lin Qi, Yi Yang: submitted to Journal of Infrared and laser engineering (2011) (In Chinese)

[2] Guifen Xia, Baojun Zhao, Qiuyue Han: submitted to Journal of Laser and infrared (2005) (In Chinese)

[3] Yamamoto M K, Fujita T, Aziz N H B A, et al: submitted to Journal of Atmospheric and Solar-Terrestrial Physics (2014)

[4] Xiangcheng Chen. Study on the Method and System of Pulsed Laser Radar Echo Treatment [D]. University of Science and Technology of China, 2015. (In Chinese)

[5] Pengge Ma, Qiuchun Jin, et al: submitted to Journal of Infrared and Laser Engineering (2012) (In Chinese)

[6] Yanhui Li, Zhenshen Wu, Yanjun Gong, et al: submitted to Acta Physica Sinica (2010) (In Chinese)

[7] Yujing Huo, Qiansong Chen, Zhiwen Pan: submitted to Journal of Infrared and laser engineering (2001) ( In Chinese) 